\title{
Endoscope-assisted mediastinal drainage therapy for anastomosis leakage after esophagectomy: a retrospective cohort study
}

\author{
Wei Guo", Lianggang Zhu\#, Yuquan Wu\#, Su Yang, Hailei Du, Xiang Zhou, Jiaming Che, Junbiao Hang, \\ Hecheng Li
}

Department of Thoracic Surgery, Ruijin Hospital, Shanghai Jiao Tong University School of Medicine, Shanghai 200025, China

Contributions: (I) Conception and design: H Li, W Guo; (II) Administrative support: J Hang; (III) Provision of study materials or patients: H Li, J Hang, J Che, L Zhu; (IV) Collection and assembly of data: Y Wu, L Zhu; (V) Data analysis and interpretation: W Guo, Y Wu; (VI) Manuscript writing: All authors; (VII) Final approval of manuscript: All authors.

\#These authors contributed equally to this work.

Correspondence to: Hecheng Li, MD, PhD. Department of Thoracic Surgery, Ruijin Hospital, Shanghai Jiao Tong University School of Medicine, Shanghai 200025, China. Email: lihecheng2000@hotmail.com.

\begin{abstract}
Background: Anastomosis leakage after esophagectomy is a major threat which leads to many subsequent complications even mortality. But current diagnosis and treatment methods are inefficient. This retrospective study aims to evaluate the utilization of endoscope-assisted mediastinal drainage therapy in treatment for anastomosis leakage after esophagectomy.

Methods: Between January 2014 and June 2018, 51 patients were confirmed anastomosis leakage using gastroscopy. Of them, 23 patients were treated with endoscope-assisted mediastinal drainage therapy (drainage group); and the other 28 patients received endoscope-assisted biomedical fibrin glue occlusion (occlusion group). Short-term clinical outcomes were examined. Factors related to length of postoperative hospitalization (LPH) was analyzed.

Results: Endoscope provided highly accurate information on the condition of anastomosis leakage. And there was no evidence that early endoscopy could cause damage to the anastomosis or gastric conduit. One patient from drainage group and two from occlusion group discharged against medical advice. Other 48 patients were completely cured without reoperation or mortality. The median LPH was 32 days in drainage group (range from 17 to 80 days) and 81 days in occlusion group (range from 32 to 190 days), respectively $(\mathrm{P}<0.05)$. Linear regression indicated statistically significant correlation between LPH and length from diagnosis to drainage or occlusion $(\mathrm{R}=0.688, \mathrm{P}<0.001)$.
\end{abstract}

Conclusions: Endoscope-assisted mediastinal drainage therapy is a satisfactory treatment for anastomosis leakage. Early diagnosis and treatment may facilitate the recovery of anastomosis leakage and reduce LPH.

Keywords: Anastomotic leakage; esophageal cancer; endoscopy; drainage; biomedical fibrin glue

Submitted Sep 21, 2019. Accepted for publication Nov 19, 2019.

doi: 10.21037/atm.2019.11.103

View this article at: http://dx.doi.org/10.21037/atm.2019.11.103

\section{Introduction}

Esophageal cancer is the eighth most common cancer worldwide, and the sixth most common cause of death from cancer (1). As the only curative treatment for resectable esophageal cancer patients, esophagectomy is associated with quite high mortality historically. Over the last few decades, the mortality after esophagectomy has decreased to $3-7 \%(2-4)$. The improvement was partly attributed to the decrease of mortality caused by anastomotic leakage (4). But till now, postoperative leakage still remains the major 
threat for esophageal cancer patients. According to previous studies, the incidence of anastomotic leakage is about $8.0-27.7 \%(5,6)$ and it results in a mortality of $3.4-6.1 \%$ $(7,8)$. Nowadays, centralization and standardization of the surgical procedure, combined with advances in perioperative care, have brought down the incidence and mortality of anastomotic leakage (9), but current therapies to treat anastomotic leakage are still inefficient. Therapy itself ranges from aggressive surgical intervention to conservative treatment such as placement of chest drains and gastrointestinal tubes, self-expanding stent treatment (10-14). Esophageal stents seem to be a promising management of anastomosis leakage (15). However, insertion of stents may result in inadequate drainage of necrotic tissue, and the dislocation rate could go up to $40 \%$ (13). If the drainage of the necrotic tissue is not sufficient, the continuous accumulation of necrosis tissue may lead to severe mediastinal infection even main artery rupture. In recent years, a variety of innovative endoscopic methods for defect closure have been developed, including endoscopy fibrin glue injection and clipping $(16,17)$.

Reasons for esophageal anastomotic leakage including the lack of serosa, shortage of circular muscle, sparse blood supply and the negative pressure from pleural space. Moreover, the tension of elevated gastric conduit may also contribute to anastomotic leaks. With regard to subsequent morbidity and mortality after anastomotic leakage, it is a general consensus that the main causes are bacterial toxin absorption, severe mediastinitis and cardiopulmonary function impairment. Therefore, keep the drainage path unobstructed and cavity clean is crucial to the healing of anastomotic leakage. Based on this concept, we designed an endoscope-assisted mediastinal drainage therapy. To further investigate the efficiency of this new method, we conducted this retrospective study.

\section{Methods}

\section{Data source and patient selection}

Between January 2014 and June 2018, a single-center institutional case series of 51 anastomosis leakage patients from 971 patients that were operated for esophageal carcinoma with gastric conduit were enrolled in this study. These esophagectomies were conducted by four surgeons (Lianggang Zhu, Jiaming Che, Junbiao Hang, Hecheng Li). All 51 patients were confirmed anastomosis leakage using gastroscopy. According to doctors' personal disposition, 23 of them received endoscope-assisted mediastinal drainage therapy (drainage group), and the other 28 patients received endoscope-assisted biomedical fibrin glue occlusion (occlusion group). Notably, drainage method is exclusively available to intrathoracic anastomosis. This study was registered at Research Registry (ID: researchregistry2324) and approved by Ruijin Hospital Ethics Committee (No. 2016-34).

All these patients were diagnosed with esophageal cancer by microscopic pathology examination preoperatively. Each patient received an esophageal contrast barium study, computed tomography (CT) of the chest and epigastrium, and abdominal ultrasonography prior to surgery. Surgical approach was determined by the tumor location. Ivor-Lewis esophagectomy is the most commonly used approach. McKeown esophagectomy and Sweet esophagectomy were also applied. Despite different surgical procedure, nasogastric tube was intubated preoperatively to apply gastrointestinal decompression in all patients. A transdermal chest tube was intubated intraoperative routinely. Another important tube was the mediastinal tube which is intraoperatively placed near the anastomosis. Jejunostomy was routinely conducted intraoperatively to maintain enteral nutrition.

Patients who were suspicious of postoperative anastomosis leakage will receive esophagography examination using meglumine diatrizoate, contrast-enhanced CT examination or methylene blue test and be confirmed by gastroscopy finally.

In drainage group, 13 patients received endoscopeassisted mediastinal drainage therapy meanwhile their very first endoscopy examination to diagnose anastomosis leakage. And the other 10 patients received this therapy after several days' symptomatic treatment, such as antibiotic therapy according to drug sensitive test. In occlusion group, it was necessary for patients to come through acute phase and get stable vital signs before endoscope-assisted biomedical fibrin glue occlusion. Symptomatic treatment was very important during this period. Application of these two methods received unanimous approval from all patients and involved surgeons.

\section{Endoscope-assisted mediastinal drainage therapy}

\section{Description of the device}

The device of mediastinal drainage therapy consisted of one naso-mediastinal tube which was intubated into the leakage fistulae, one mediastinal tube nearby the anastomosis which was intraoperatively placed routinely, one chest tube, and the infusion equipment with or without vacuum aspiration 


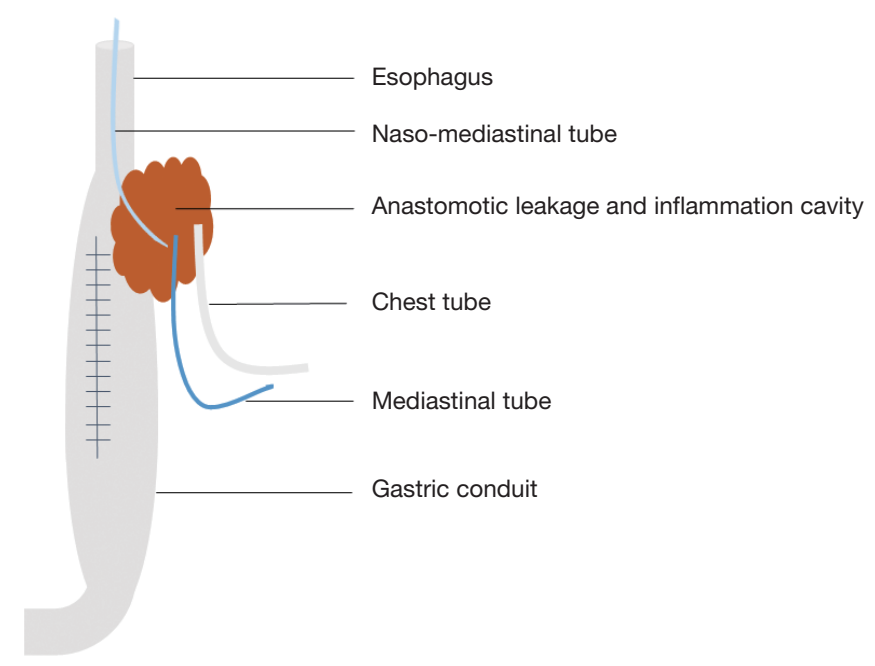

Figure 1 Schematic diagram of the endoscope-assisted mediastinal drainage therapy.

(Figure 1). The naso-mediastinal tube is placed through the crevice of the anastomosis leakage under the assistance of gastroscopy after extubation of nasogastric tube (Figure $2 A, B$ ).

Before the placement of this nasogastric tube, we tie a knot in the end of the tube so that the gastroscope could handle the tube conveniently. The diameter of the tube is determined by the diameter of the anastomotic crevice. In this study, the diameter of the anastomotic crevice ranges from 8 to $18 \mathrm{~mm}$, the common gastric tube fit well in most patients. Naso-mediastinal tube is stuck on the patient's nose by a wing-shaped sticky tape and is fixed by a rope around his head. Normal saline continuously flush into the inflammation cavity through the naso-mediastinal tube, to rinse the necrosis tissue outflow through the chest tube and mediastinal tube.

Figure 2 shows the intubation of naso-mediastinal tube and its efficiency in a 61 years old male patient. He received minimally invasive Ivor-Lewis esophagectomy at August $1^{\text {st }}$ 2017. At $8^{\text {th }}$ postoperative day (POD) he was diagnosed with anastomosis leakage after CT examination (Figure 2C,D). $\mathrm{He}$ therefore received endoscope-assisted mediastinal drainage therapy at the very next day. After 7 days drainage, his vomica got obviously eliminated as shown in the CT image (Figure 2E,F).

\section{Drainage}

Drainage is the critical procedure for the treatment of anastomotic leakage. Relative study indicated that the mortality of patients whose anastomotic leak is insufficiently drained could up to $80 \%$ (13). Historically, surgical revision is necessary if percutaneous drainage is impractical or not effective. But using our method, it is effective and minimally invasive to expel the contaminative fluid from leakage cavity. Mediastinal drainage was first mentioned by Dr. Weidenhagen (18), after that more studies revealed the efficiency of this method $(15,16)$. Our drainage method is quite different from theirs because we believe that adequate flushing could promote the circulating out of pus and septic. So, we use an infusion equipment to lavage the cavity constantly and drain the contaminative fluid through the vacuum extractor simultaneously. There is no strict limitation which tube is for transfusion and which one for drainage. Normally, we transfused normal saline through the naso-mediastinal tube and drain from the mediastinal tube and chest tube. In some cases, the patients' chest tube was pulled out before the diagnosis of anastomosis leakage. For these patients, the mediastinal tube itself could still drained well.

At first the symptoms of infection are obvious, the lavage speed could be $100 \mathrm{~mL} / \mathrm{h} \times 24 \mathrm{~h}$. Along with the patient's condition getting better, the lavage volume and transfusion duration should be turned down accordingly to promote healing of the anastomosis. From then on, we can also drain manually from the naso-mediastinal tube using syringe whenever this tube is not occupied.

\section{Tube drawing}

When patients' vital sighs get stable according to body temperature, leukocyte count and CT scan results, we consider pulling up the tubes. Firstly, we pull out the 

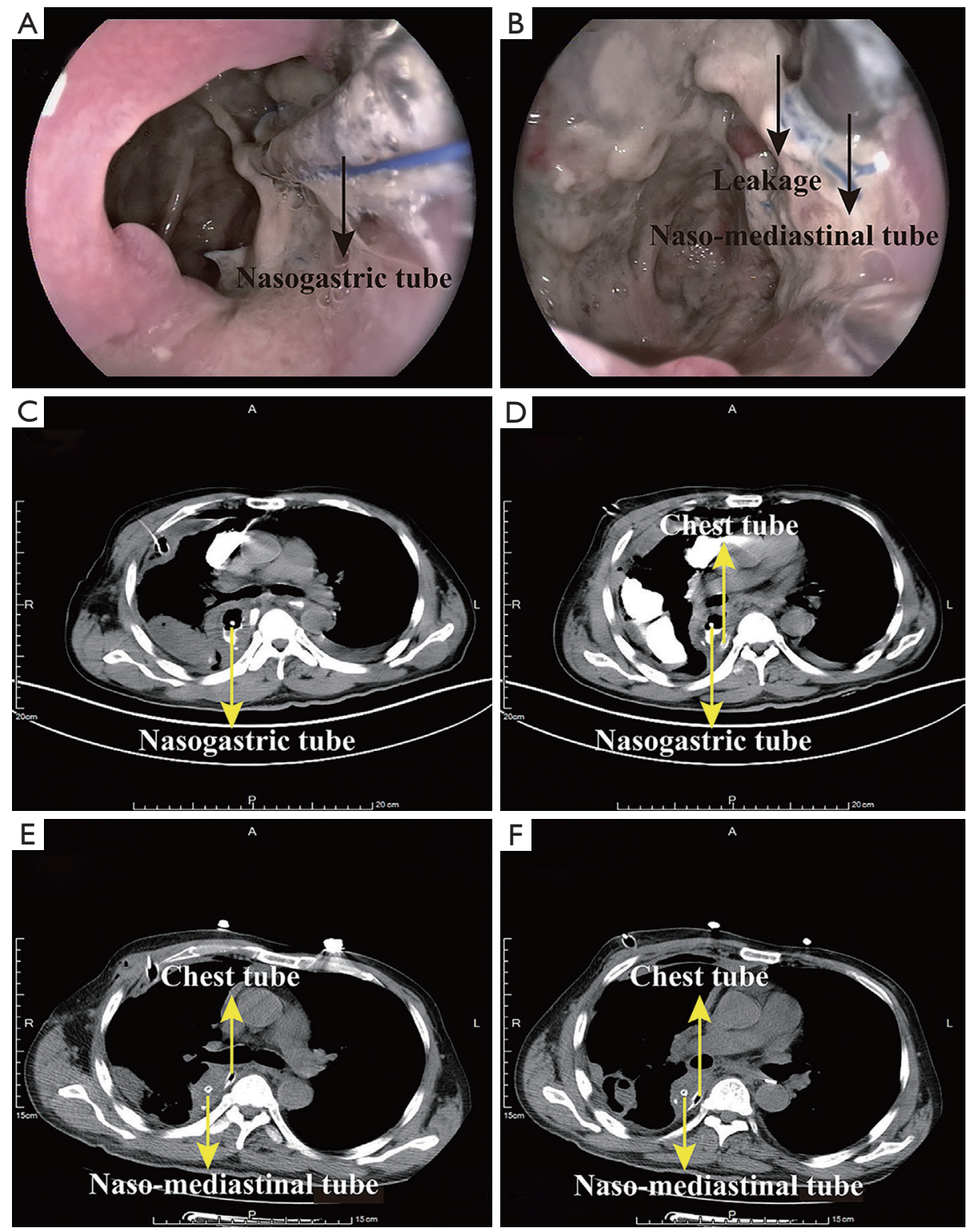

Figure 2 Intubation of the naso-mediastinal tube and its efficiency. (A) Extubation of nasogastric tube; (B) intubation of naso-mediastinal tube; (C,D) CT images before drainage; (E,F) CT images after 7 days drainage. CT, computed tomography.

naso-mediastinal tube and chest tube so that the necrosis tissue could still drain out well. Then we pull out the mediastinal tube $1-2 \mathrm{~cm}$ per day so that the residual necrosis could drain out completely. Every time we pull out the mediastinal, it is necessary to cut off the lengthy tube and use a paper clip pierce through the mediastinal tube lest the tube retract into chest. Adhesive bandages are used to prevent topical infection and abjunction of the mediastinal tube.

\section{Endoscope-assisted biomedical fibrin glue occlusion}

Description of the device

To fulfill the occlusion, OlypumsGIF-160 electronic gastroscope or OlypumsGIF-XQ260 electronic gastroscope, medical dual-lumen catheter, syringe and biomedical fibrin glue are needed. Depending on the leakage diameter, it may need one to three set of medical dual-lumen catheter, syringe and biomedical fibrin glue. Fibrin glue is a sealant, two-component topical hemostat, and tissue adhesive 

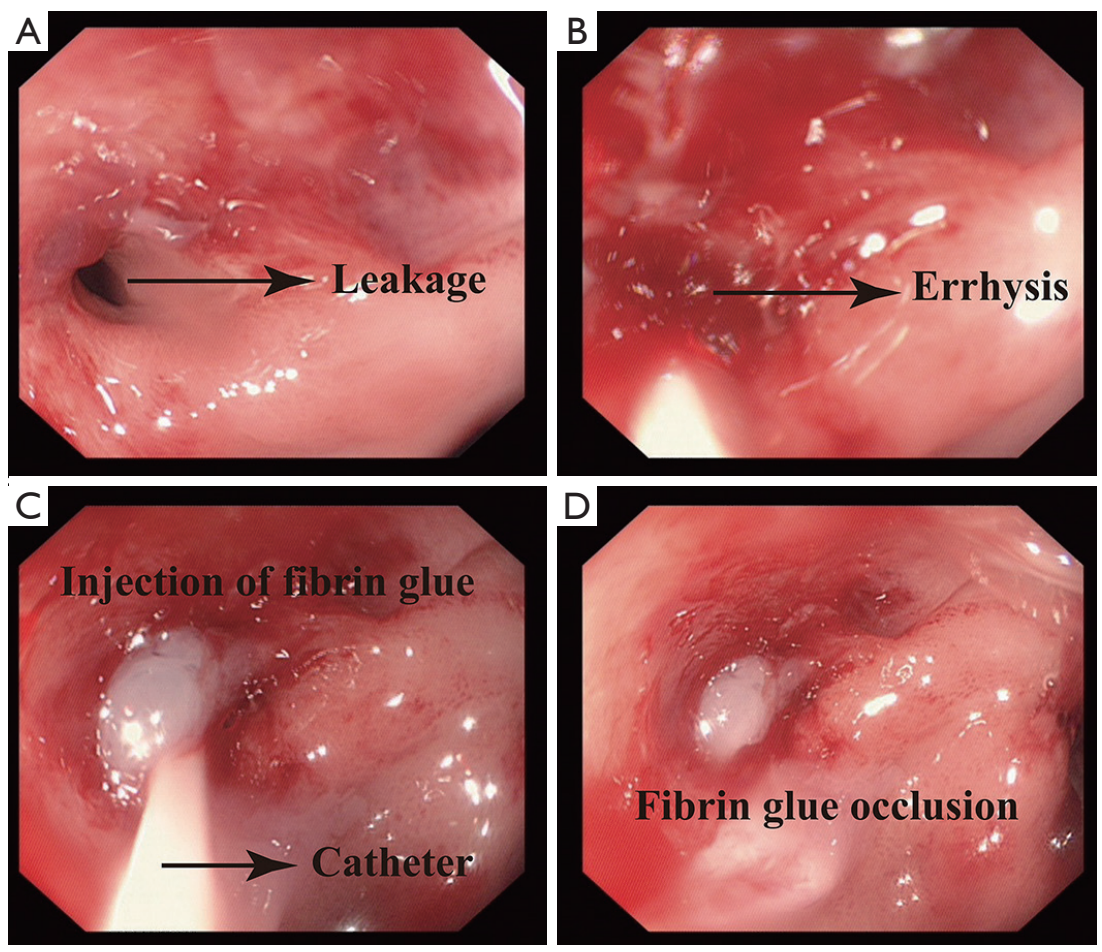

Figure 3 Procedure of biomedical fibrin glue occlusion. (A) Exposing and cleaning of the leakage; (B) rubbing the edge of the leakage, making it errhysis to facilitate granulation; (C) insertion of the dual-lumen catheter into the leakage and injection of the fibrin glue; (D) fibrin glue occlusion.

consisting of fibrinogen and thrombin $(19,20)$.

\section{Endoscope-assisted occlusion}

This therapy generally applied in patients whose leakage diameter $\leq 1 \mathrm{~cm}$ and systemic condition was stable. Usually, this operation is conducted 1 month after the diagnosis of leakage confirmed. During this period, we use enteral and parenteral nutrition and symptomatic treatment to control symptom. The patient should be fasting one day before and after the operation. Furthermore, in consideration of mediastinal abscess would not eliminate through the esophagus after occlusion, this therapy is not suitable for the patients who has too much abscess.

Step one, we expose the leakage, wash the fistula and residual cavity under the assistance of gastroscope (Figure $3 A$ ). Then we use a brush rubbing the edge of the leakage, making it errhysis to facilitate granulation (Figure 3B). At last we insert the dual-lumen catheter into the leakage (Figure $3 C$ ), inject the fibrin glue into the sinus until the whole sinus blocked (Figure $3 D$ ). An esophagography is needed to evaluate the occlusion effect 1 week later. Some patients may need three to five times occlusion.

\section{Symptomatic treatment}

These two therapies share the same symptomatic treatment. Antibiotic therapy is used to resist infection according to the patient's drug sensitive test. Isocaloric enteral nutrition is given using jejunal feeding tube. For patients with low oxygen saturation, oxygen inhalation is necessary.

\section{Statistical analysis}

Chi-square and Fisher tests were performed for categorical variables. Student's $t$-test was performed to compare two continuous variables conformed to normal distribution. For continuous variables did not conformed to normal distribution, Mann-Whitney U test was performed. Analysis of covariance (AONCA) was conducted to eliminate confounding factors. Linear regression and fitting curves were conducted to analyze the correlation between two continuous variables. Statistical analysis was performed with SPSS ${ }^{\circledR}$ version 19 (SPSS, Inc., Chicago, IL, USA) or R studio (version 1.1.463, Boston, MA, USA) and confidence interval (CI) analysis version 2.2.0 (T Bryant, University of 
Table 1 Characteristics of patients

\begin{tabular}{|c|c|c|c|}
\hline Characteristics & Drainage group & Occlusion group & $P$ value \\
\hline Male & 21 & 25 & $0.595^{\star}$ \\
\hline Female & 2 & 3 & \\
\hline Age, years & $48-73$ & $51-75$ & $0.237^{\#}$ \\
\hline Smoking history & 19 & 23 & $1.000^{\star}$ \\
\hline Comorbidity & 5 & 8 & $0.749^{\star}$ \\
\hline Tumor location & & & $0.590^{*}$ \\
\hline Middle & 5 & 4 & \\
\hline Surgical operation & & & $0.056^{*}$ \\
\hline McKeown & 0 & 3 & \\
\hline Ivor-Lewis & 23 & 20 & \\
\hline Sweet & 0 & 5 & \\
\hline Pathological staging & & & $1.000^{*}$ \\
\hline I & 2 & 3 & \\
\hline II & 12 & 14 & \\
\hline III & 9 & 11 & \\
\hline
\end{tabular}

*, chi-square test, Fisher exact test; ${ }^{\#}$, student's $t$-test.

Southampton, England). A P value $<0.05$ was considered statistic significant.

\section{Results}

A total of 51 patients with anastomotic leakage were enrolled in this study. Patients characteristics and clinical outcomes are shown in Tables 1,2, respectively. In drainage group, anastomotic leakage occurred between $5^{\text {th }}$ POD and $14^{\text {th }}$ POD (median $10^{\text {th }}$ POD). The median length from surgery to drainage was 11 (range from 5 to 33 ) days. Median length of LPH was 32 (range from 17 to 80) days. In occlusion group, anastomotic leakage occurred between $2^{\text {nd }}$ POD and $34^{\text {th }}$ POD (median $11^{\text {th }}$ POD). The median duration from surgery to occlusion was 48 (range from 27 to 100) days. Median length of LPH was 81 (range from 32 to 190) days. One patient from drainage group and two from occlusion discharged against medical advice.

Drainage group showed shorter length from diagnosis to treatment $(\mathrm{P}<0.001)$, shorter length from surgery to treatment $(\mathrm{P}<0.001)$, and shorter length of postoperative hospitalization $(\mathrm{LPH})(\mathrm{P}<0.001)$. To figure out the difference of LPH between these two groups was caused by the treatment method itself or difference of length from diagnosis to treatment, ANOCA was conducted. ANOCA result also showed decreased LPH in drainage group (Table 3).

To analyze the correlative factors with $\mathrm{LPH}$, we conducted linear regression between LPH and age, surgery to diagnosis duration, diagnosis to treatment duration, surgery to treatment duration and length after treatment. Statistical result (Table 4, Figure 4) shows a significant correlation between LPH and length from diagnosis to treatment in all patients (adjusted $\mathrm{R}=0.688, \mathrm{P}<0.001$ ). Linear regression between LPH and length from diagnosis to treatment in drainage group (adjusted $\mathrm{R}=0.336, \mathrm{P}=0.064$ ) and occlusion group (adjusted $\mathrm{R}=0.356, \mathrm{P}=0.035$ ) is shown in Figure 5 and Table 4. 
Table 2 Clinical outcomes and curative efficacy

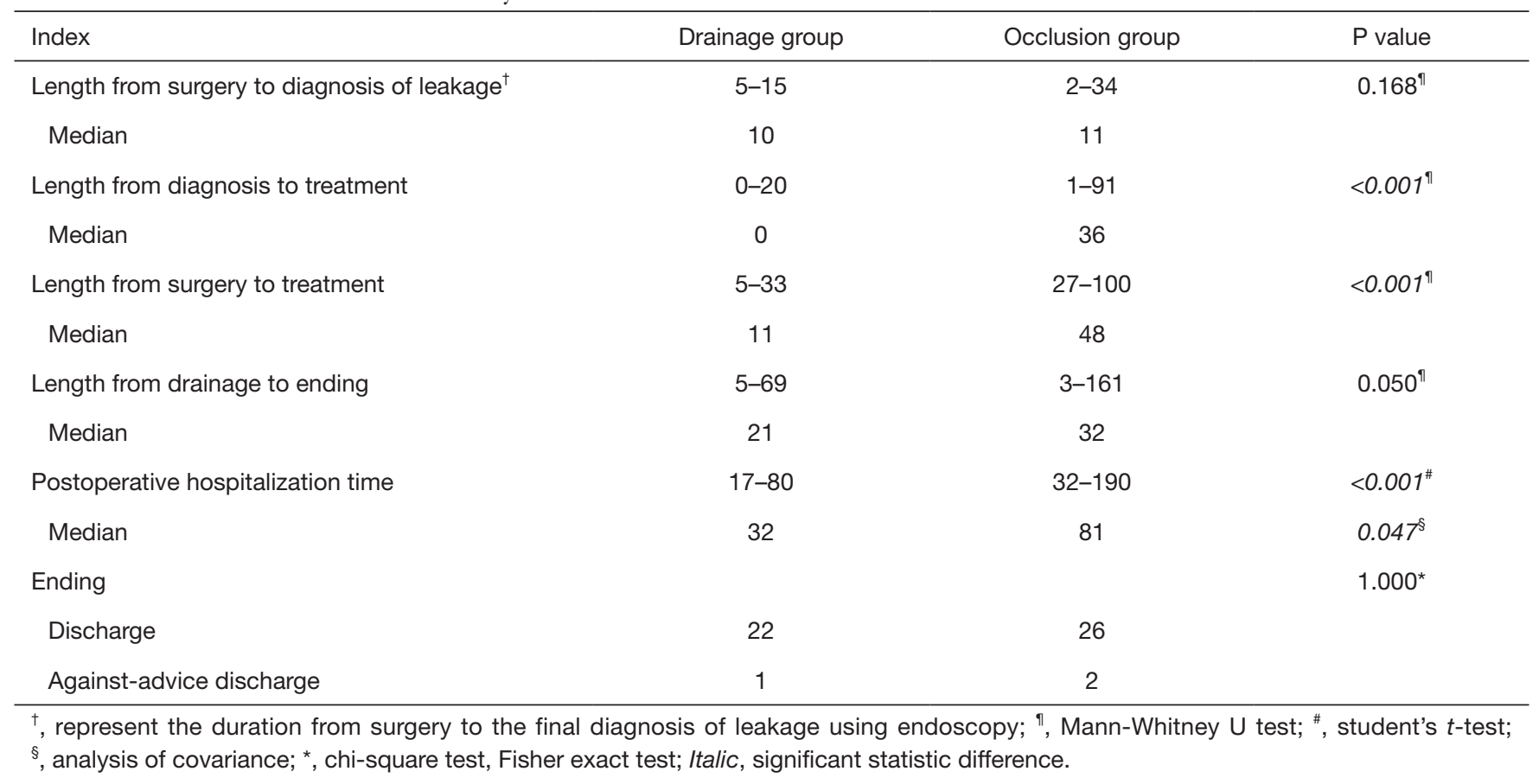

Table 3 Comparative analysis of postoperative hospitalization time of drainage group and occlusion group using $t$-test and ANCOVA

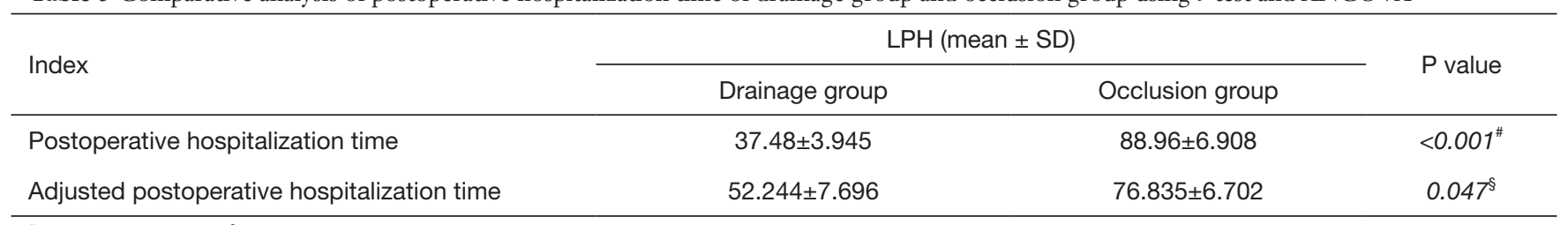

" , student's $t$-test; ${ }^{\S}$, analysis of covariance; Italic, significant statistic difference. LPH, length of postoperative hospitalization; SD, standard deviation; ANCOVA, analysis of covariance.

Table 4 Linear regression between LPH and length from diagnosis to treatment

\begin{tabular}{lccc}
\hline Group & Adjusted R & Durbin-Waston & P value \\
\hline All patients & 0.688 & 1.735 & $<0.001$ \\
Drainage group & 0.336 & 1.035 & 0.064 \\
Occlusion group & 0.356 & 1.99 & 0.035 \\
\hline
\end{tabular}

Italic, significant statistic difference.

\section{Discussion}

Anastomosis leakage remains the main reason for prolonged hospitalization time and high mortality rate of esophageal patients (4). Consequently, it is very important to make early diagnosis and take effective measures. Endoscopy proved its crucial role in diagnosis and treatment for anastomosis leakage in this study.

Digestive tract perforation is generally regarded as the contraindication for endoscopy (21). However, in this study, early endoscopy never caused complications. A recent study reported a CT-based scoring system for diagnosis of anastomotic leakage, with the sensitivity of $80 \%$ and specificity of $84 \%$, which is still not satisfactory (22). 


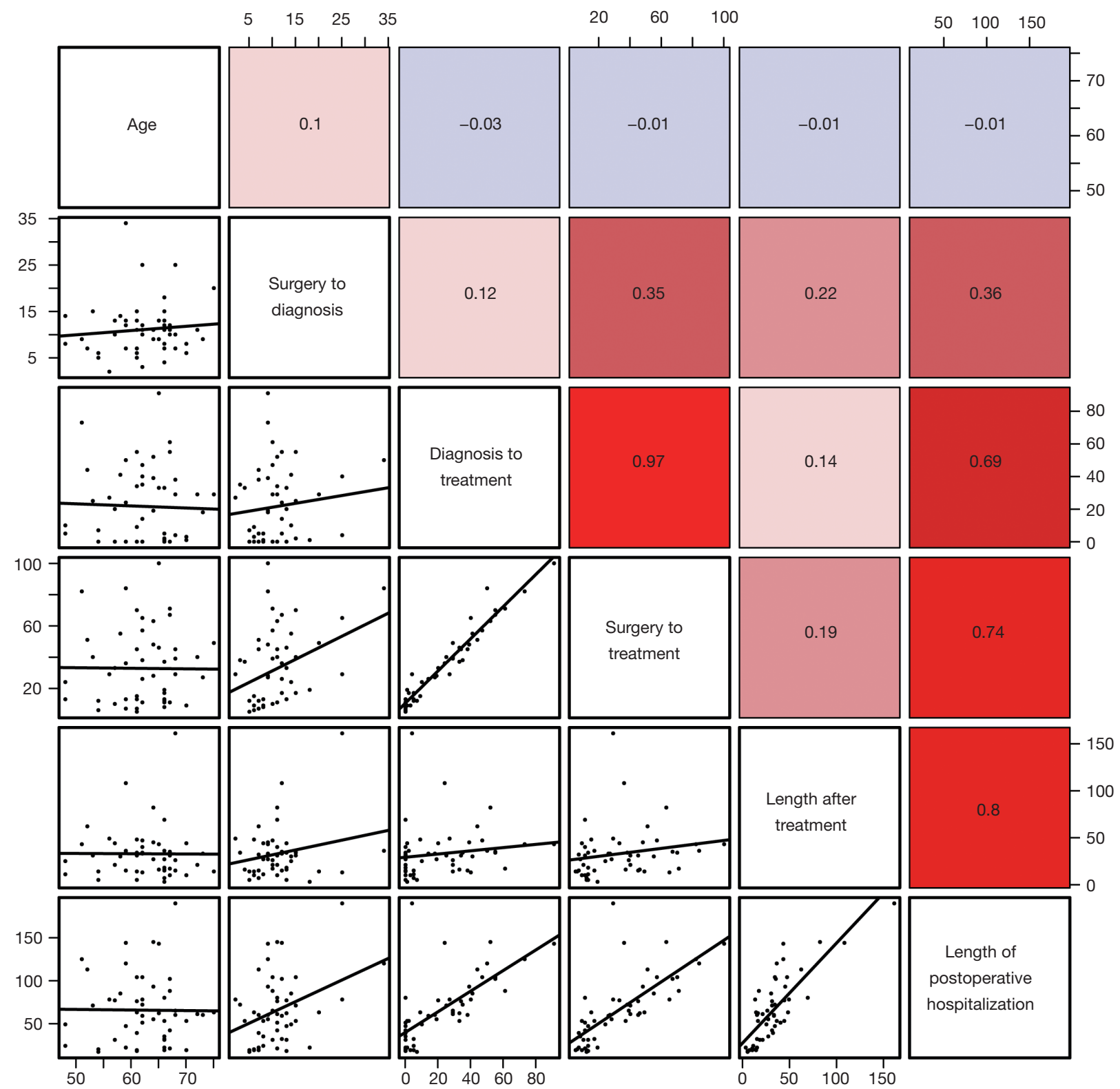

Figure 4 Correlations among age, surgery to diagnosis duration, diagnosis to treatment duration, surgery to treatment duration, length after treatment and LPH in all 51 patients. LPH, length of postoperative hospitalization.

Furthermore, compared with swallow fluoroscopy, methylene blue injection, or CT scan, endoscopy could provide extremely useful information about the condition of esophagogastric anastomosis leakage and gastric conduit, which allows individualized subsequent managements for these patients, such as mediastinal drainage or fibrin glue occlusion.

The principles of management of anastomosis leakage include drainage of pleural and mediastinal cavities, intravenous broad-spectrum antibiotics, gastric decompression, and enteral or parenteral nutritional support. Control of the abscess is essential for treating patients with anastomotic leakages. Only eradication of the infectious tissue enables the anastomosis to recover. But management of this complication still remains controversial $(16,23)$, some surgeons prefer conservative methods and some prefer reoperation. According to published data, mortality of patients who received reoperation is pretty 

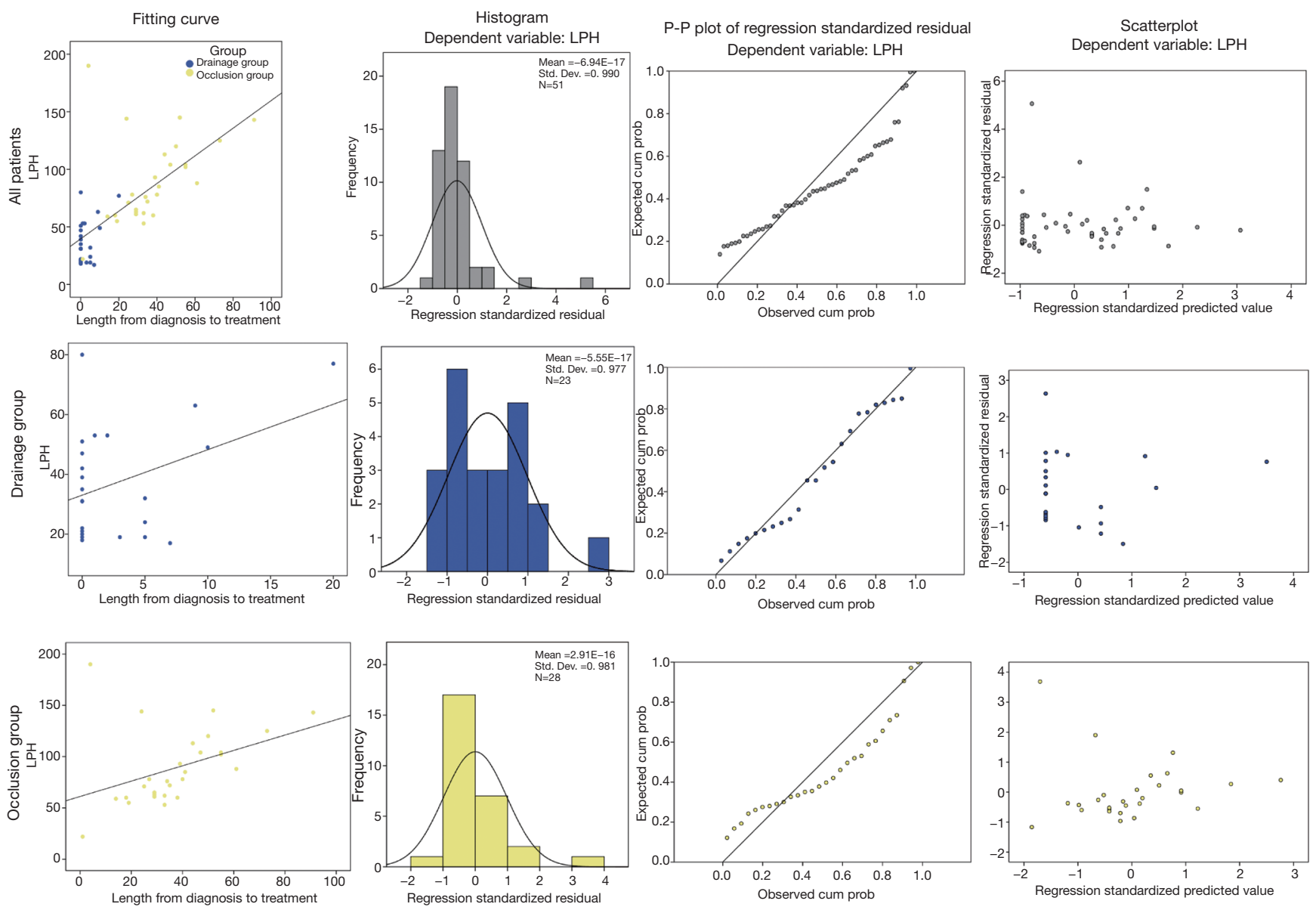

Figure 5 Linear regression between LPH and length from diagnosis to treatment in drainage and occlusion group. LPH, length of postoperative hospitalization; Std. Dev., standard deviation.

high (24). Therefore, it is necessary to establish an effective method with less trauma to heal this morbidity. Our method provides a minimally invasive and satisfactory method to accelerate necrosis discharge and leakage healing.

In our study, the transluminal system allows an effective and continuous lavage and drainage of the abscess cavity without additional trauma to the patient. And because of the naso-mediastinal tube, fluid leak from the esophagus or stomach into the cavity is partially blocked. Compared with other similar methods $(25,26)$, our method provided lower mortality and shorter hospitalization time. This improvement was attributed to the application of lavage through the whole inflammation cavity, which accelerated the drainage of necrosis tissue.

The fibrin glue occlusion therapy was generally applied in patients whose leakage diameter $\leq 1 \mathrm{~cm}$ and systemic condition was stable. In this group, LPH was apparently longer than drainage group. This was partly because we can deal with the leakage immediately as soon as we diagnose the leakage in drainage group, but in occlusion group we have to wait about 1 month till the patients pass through acute phase. In addition, using the circulatory mediastinal vacuum drainage therapy we could clean up the necrotic tissue rapidly and radically. Compared with drainage group, LPH in occlusion group was significantly longer, partially confirmed the importance of drainage in leakage patients. However, endoscope-assisted biomedical fibrin glue occlusion also has its own advantage. For the patients whose leakage is small and clinical symptoms is not so severe, using fibrin glue occlusion does not need additional tube and do not affect their normal activity.

This study first revealed the significant correlation between length from diagnosis and LPH via linear regression analysis, which manifested the importance of 
early diagnosis and treatment for anastomosis leakage.

Various limitations apply to this study. First, this study is limited by its small sample size. Second, heterogeneity between patients may influence the final results. Further clinical trials are needed to confirm our study.

In conclusion, our study indicated that endoscopeassisted mediastinal drainage therapy is safe and effective for patients with anastomotic leakage. Early diagnosis and treatment for anastomosis leakage are important for reducing patients' hospitalization time.

\section{Acknowledgments}

Funding: This study was supported by the grant from Science and Technology Commission of Shanghai Municipality Medical Guidance Science\& Technology Support Project (16411966100), Shanghai Municipal Education Commission- Gaofeng Clinical Medicine Grant Support (20172005) and Shanghai Municipal Commission of Health and Family Planning Outstanding Academic Leaders Training Program (2017BR055). The funding body had no role in the design of the study and collection, analysis, and interpretation of data and in writing the manuscript.

\section{Footnote}

Conflicts of Interest: The authors have no conflicts of interest to declare.

Ethical Statement: The authors are accountable for all aspects of the work in ensuring that questions related to the accuracy or integrity of any part of the work are appropriately investigated and resolved. This study was registered at Research Registry (ID: researchregistry2324) and approved by Ruijin Hospital Ethics Committee (NO. 2016-34).

\section{References}

1. Torre LA, Bray F, Siegel RL, et al. Global cancer statistics, 2012. CA Cancer J Clin 2015;65:87-108.

2. Fuchs HF, Harnsberger CR, Broderick RC, et al. Mortality after esophagectomy is heavily impacted by center volume: retrospective analysis of the Nationwide Inpatient Sample. Surg Endosc 2017;31:2491-7.

3. Guo W, Yang S, Li H. Esophagectomy with gastric conduit reconstruction for benign disease: extreme but important. Ann Transl Med 2018;6:117.

4. Lerut T, Coosemans W, Decker G, et al. Surgical techniques. J Surg Oncol 2005;92:218-29.

5. Kassis ES, Kosinski AS, Ross P Jr, et al. Predictors of anastomotic leak after esophagectomy: an analysis of the society of thoracic surgeons general thoracic database. Ann Thorac Surg 2013;96:1919-26.

6. Goan YG, Chang HC, Hsu HK, et al. An audit of surgical outcomes of esophageal squamous cell carcinoma. Eur J Cardiothorac Surg 2007;31:536-44.

7. Kawoosa NU, Dar AM, Sharma ML, et al. Transthoracic versus transhiatal esophagectomy for esophageal carcinoma: experience from a single tertiary care institution. World J Surg 2011;35:1296-302.

8. Safranek PM, Sujendran V, Baron R, et al. Oxford experience with neoadjuvant chemotherapy and surgical resection for esophageal adenocarcinomas and squamous cell tumors. Dis Esophagus 2008;21:201-6.

9. Guo W, Ma L, Zhang Y, et al. Totally minimally invasive Ivor-Lewis esophagectomy with single-utility incision video-assisted thoracoscopic surgery for treatment of midlower esophageal cancer. Dis Esophagus 2016;29:139-45.

10. Dai YY, Gretschel S, Dudeck O, et al. Treatment of oesophageal anastomotic leaks by temporary stenting with self-expanding plastic stents. Br J Surg 2009;96:887-91.

11. Rutegård $M$, Lagergren $P$, Rouvelas I, et al. Intrathoracic anastomotic leakage and mortality after esophageal cancer resection: a population-based study. Ann Surg Oncol 2012;19:99-103.

12. Hoeppner J, Kulemann B, Seifert G, et al. Covered selfexpanding stent treatment for anastomotic leakage: outcomes in esophagogastric and esophagojejunal anastomoses. Surg Endosc 2014;28:1703-11.

13. Kauer WK, Stein HJ, Dittler HJ, et al. Stent implantation as a treatment option in patients with thoracic anastomotic leaks after esophagectomy. Surg Endosc 2008;22:50-3.

14. Tuebergen D, Rijcken E, Mennigen R, et al. Treatment of thoracic esophageal anastomotic leaks and esophageal perforations with endoluminal stents: efficacy and current limitations. J Gastrointest Surg 2008;12:1168-76.

15. Dasari BV, Neely D, Kennedy A, et al. The role of esophageal stents in the management of esophageal anastomotic leaks and benign esophageal perforations. Ann Surg 2014;259:852-60.

16. Turkyilmaz A, Eroglu A, Aydin Y, et al. The management of esophagogastric anastomotic leak after esophagectomy for esophageal carcinoma. Dis Esophagus 2009;22:119-26.

17. Kirschniak A, Subotova N, Zieker D, et al. The over-the- 
scope clip (OTSC) for the treatment of gastrointestinal bleeding, perforations, and fistulas. Surg Endosc 2011;25:2901-5.

18. Weidenhagen R, Hartl WH, Gruetzner KU, et al. Anastomotic leakage after esophageal resection: new treatment options by endoluminal vacuum therapy. Ann Thorac Surg 2010;90:1674-81.

19. Spotnitz WD. Fibrin sealant: past, present, and future: a brief review. World J Surg 2010;34:632-4.

20. Nakano Y, Takao T, Morita Y, et al. Endoscopic plombage with polyglycolic acid sheets and fibrin glue for gastrointestinal fistulas. Surg Endosc 2019;33:1795-801.

21. ASGE Standards of Practice Committee, Early DS, BenMenachem T, et al. Appropriate use of GI endoscopy. Gastrointest Endosc 2012;75:1127-31.

22. Goense L, Stassen PMC, Wessels FJ, et al. Diagnostic performance of a CT-based scoring system for diagnosis of anastomotic leakage after esophagectomy:

Cite this article as: Guo W, Zhu L, Wu Y, Yang S, Du H, Zhou X, Che J, Hang J, Li H. Endoscope-assisted mediastinal drainage therapy for anastomosis leakage after esophagectomy: a retrospective cohort study. Ann Transl Med 2019;7(23):747. doi: 10.21037/atm.2019.11.103 comparison with subjective CT assessment Eur Radiol 2017;27:4426-34.

23. Semenkovich TR, Meyers BF. Surveillance versus esophagectomy in esophageal cancer patients with a clinical complete response after induction chemoradiation. Ann Transl Med 2018;6:81.

24. Eizaguirre E, Larburu S, Asensio JI, et al. Treatment of anastomotic leaks with metallic stent after esophagectomies. Dis Esophagus 2016;29:86-92.

25. Schniewind B, Schafmayer C, Voehrs G, et al. Endoscopic endoluminal vacuum therapy is superior to other regimens in managing anastomotic leakage after esophagectomy: a comparative retrospective study. Surg Endosc 2013;27:3883-90.

26. Schorsch T, Müller C, Loske G. Endoscopic vacuum therapy of anastomotic leakage and iatrogenic perforation in the esophagus. Surg Endosc 2013;27:2040-5. 